This is the final peer-reviewed accepted manuscript of:

Casari, M., \& Dragone, D. (2015). Choice reversal without temptation: A dynamic experiment on time preferences. Journal of Risk and Uncertainty, 50(2), 119-140

The final published version is available online at:

https://doi.org/10.1007/s11166-015-9211-x

Rights / License:

The terms and conditions for the reuse of this version of the manuscript are specified in the publishing policy. For all terms of use and more information see the publisher's website. 


\title{
Choice Reversal without Temptation: A Dynamic Experiment on Time Preferences*
}

\author{
Marco Casari ${ }^{\dagger} \quad$ Davide Dragone
}

March 5, 2015

\begin{abstract}
We study intertemporal choices through an experiment run over multiple dates and we show that intertemporal behavior is affected by additional drivers beyond impatience and present-biased preferences. By eliciting a subject's plan and tracking its implementation over time, this dynamic design helps our understanding of time inconsistency through the identification and measurement of three notions of choice reversal in intertemporal behavior. In the experiment, there is widespread choice reversal and demand for flexibility. Neither the usual exponential nor hyperbolic discounting models can account for the observed behavior.
\end{abstract}

JEL Classifications: C91, D01, D80, D90

${ }^{*}$ An earlier draft circulated with the title "Impatience, Anticipatory Feelings, and Uncertainty: A Dynamic Experiment on Time Preferences". We thank for comments Alberto Bisin, Tim Cason, Luigi Franzoni, Giuseppe Pignataro and the participants at M-BEES 2010 in Maastricht, EWEBE 2010 in Bertinoro, IMEBE 2011 in Barcelona, BEE 2011 in Florence, SAET 2011 in Faro, Max Planck Institute of Economics in Jena, Purdue University and University of Bologna. We also thank the composer Paolo Ingrosso for providing the noise for the experiment. This paper is part of the SONIC project (RBFR084L83-FIRB) at the University of Bologna. Financial support from AICCON, from the Italian Government (PRIN 2007/MCKEYA_003), and from the Max Planck Institute of Economics in Jena (ESI) is gratefully acknowledged. The usual disclaimer applies.

${ }^{\dagger}$ Corresponding author: Marco Casari, Department of Economics, University of Bologna, Piazza Scaravilli 2, 40126, Bologna, Italy; marco.casari@unibo.it.

${ }^{\ddagger}$ Department of Economics, University of Bologna, Piazza Scaravilli 2, 40126, Bologna, Italy; davide.dragone@unibo.it. 
Keywords: longitudinal experiment, time inconsistency, self-control, risk, real-effort experiment 
Intertemporal choices are ubiquitous in economics and understanding their motivations is crucial for predicting patterns of retirement savings, purchasing of durable goods, addictions and health choices, just to name a few. The empirical literature has shown that impatience and temptation are major motivations of intertemporal choices (e.g., Schelling, 1978, Warner and Pleeter, 2001, Benartzi and Thaler, 2004, Brown et al., 2009, Tanaka et al., 2010). In addition, there may be other important drivers such as risk, uncertainty, and anticipatory feelings (Loewenstein and Prelec, 1991, Sozou, 1998, Fernandez-Villaverde and Mukherji, 2002, Dasgupta and Maskin, 2005, Halevy, 2008, Manzini and Mariotti, 2010). Consider for instance a farmer who is making plans for the following year and can choose between a high-yield crop and a dry-resistant crop. Given the farmer's experience of past harvests and precipitations, the high-yield crop is ex-ante preferred. Before the spring comes, though, the farmer realizes that the coming year will be unusually dry, so he rationally switches to the dry-resistant crop. Any ex-post deviation from the initial plan denotes choice reversal, but this one does not originate from time inconsistent preferences; rather it springs from weather uncertainty. The farmer has simply taken advantage of updated information.

The empirical challenge is how to design an experiment that can disentangle the role of impatience and temptation from the role played by other drivers which are still relevant for intertemporal behavior. To this end, we designed and ran an experiment of intertemporal choices in a dynamic setting where subjects choose at multiple dates and impatient agents are predicted to behave in a similar way, irrespective of whether they are exponential or quasi-hyperbolic discounters. Hence, observed deviations from these predictions ought to originate from motivations other than impatience or temptation. In the experiment, subjects faced a real-effort task to be completed under a deadline. The task consisted in listening to an annoying noise in the lab for twenty minutes which, in a sense, is prototypical of any activity requiring a costly effort. Each subject attended three sessions in exchange for a fixed participation fee. For each session, the basic elements of the experimental design were as follows.

In Session 0: Subjects had to rank three options: to listen to the noise immediately $(t=0)$, in two weeks $(t=1)$ or in four weeks $(t=2)$. In addition, subjects bid in an auction to acquire the possibility of revising their ranking in two weeks, i.e. they could buy flexibility. All choices were properly incentivized.

\footnotetext{
${ }^{1}$ Using noise as a stimulus is common among experimental psychologists (e.g., Millar and Navarick, 1984).
} 
In Session 1: All subjects who had not listened to the noise in Session 0 had to rank two options: to listen to the noise immediately $(t=1)$ or in two weeks $(t=2)$. The ranking elicited in Session 1 applied if the subject had won the auction to revise her ranking; otherwise, the ranking elicited in Session 0 applied.

In Session 2: All subjects who had not listened to the noise in either Session 0 or Session 1 listened to the noise.

The dynamic design of the experiment allows for a deeper understanding of intertemporal behavior and time inconsistency, both on theoretical and empirical grounds. It is well known that, under the customary assumption of exponential time preferences, an individual chooses an optimal plan of consumption which, given the same information, will be carried out without deviations (Strotz, 1955). To validate this prediction, one should follow the individual behavior over time or, at least, elicit the current plan and then track its future implementation. However, many experiments adopt a different technique in order to study time consistency: they compare a series of intertemporal choices elicited at a given moment. This cross-sectional design is essentially static, because subjects make all their choices at one date. In contrast, we adopted a longitudinal experimental design where individuals make decisions at multiple dates (Sayman and Öncüler, 2009, Read et al., 2012) 2 This dynamic design provides direct evidence on time inconsistency and self-control problems, and it also allows us to identify and measure different complementary notions of choice reversal over time.

Employing a good to be "consumed" in the lab rather than money and using it as a stimulus for eliciting incentive-compatible choices is an additional distinctive feature of our experimental design. It yields two main advantages. First, it solves some methodological issues in the empirical measurement of time preferences, in particular storability and tradability of the reward, which potentially affect experiments using monetary payments. Second, and more importantly, since individual preferences are defined over consumption goods, this design provides direct evidence on intertemporal preferences and it allows for a better understanding of the underlying drivers.

Although the task was not pleasurable, about two thirds of the experimental subjects chose to complete it immediately. This result cannot be explained by considering discounting

\footnotetext{
${ }^{2}$ Other authors have used different terms to address the same distinction. See, e.g., Read et al. (1999) and references therein, and Frederick et al. (2002).
} 
as the only driver of intertemporal choices. Moreover, the experiment was designed in such a way that temptation alone could not cause any choice reversal, and yet choice reversal was common among the subjects. This evidence shows the relevance of other motivations for intertemporal choices beyond impatience and temptation, such as, for instance, uncertainty and new information.

We draw two main conclusions. First, impatience is an important factor for intertemporal decision making, but a model based on discounting as the only driver of intertemporal choice can have a low descriptive and predictive power. Second, uncertainty about future utility most likely plays a substantial role. The explicit inclusion of uncertainty when modelling intertemporal choice and interpreting the experimental data will substantially improve our understanding of the mechanisms driving people's behavior over time. It would also suggest new ways to cope with time inconsistency and self-control issues, on which there is substantial evidence in the field, because policies that supply precommitment mechanisms for presentbiased people may work differently if uncertainty plays a role. In this direction, a promising venue for future research is the design and analysis of policies that affect the uncertainty people are subject to.

The paper is structured as follows. Section 1 reviews the literature. Section 2 describes the experimental design. Section 3 describes the theoretical setup and introduces three notions of choice reversal that can be measured through a dynamic setup. Section 4 advances theoretical predictions for models driven by discounting and by uncertainty. Section 5 presents the experimental results. Discussions and concluding remarks are presented in Sections 6 and 7.

\section{Literature review}

The Discounted Utility model (Samuelson, 1937) is the most popular model for studying intertemporal choices. Under exponential discounting and additive separability, the model predicts the future to be discounted at a constant rate, which precludes the possibility of time-inconsistent behavior (Strotz, 1955). To test this prediction, in most experimental studies subjects are required to solve intertemporal trade-offs by choosing between goods or monetary amounts available at different future dates. The typical design requires subjects to go to the lab only once, and the whole elicitation process is completed in that occasion 
(Kirby, Herrnstein, 1995). Only a minority of experiments are dynamic in the sense that they track individual choices over multiple dates (for a similar point, see also Sayman and Öncüler, 2009). Studies with a dynamic design have focused on real-effort tasks, as well as on monetary choices, and provide direct evidence on how subjects implement their plan of action as time goes on. For example, Ainslie and Haendel (1983) focus on substance abuse patients, Read and van Leeuwen (1998) study the choice between healthy and unhealthy snacks, Read et al. (1999) ask subjects to choose between highbrow and lowbrow rental movies, Ariely and Wertenbroch (2002) consider studying activities, Dellavigna and Malmendier (2006) and Charness and Gneezy (2009) focus on choices concerning attendance at the gym and physical exercise. In the recent literature, a dynamic experimental design with monetary incentives has been used in Casari (2009), Sayman and Öncüler (2009), Ginè et al. (2012), and Read et al. (2012).

A robust finding in the literature on intertemporal behavior is that people are often subject to present-biased preferences and to choice reversals. To account for this evidence, many scholars argue in favor of a quasi-hyperbolic model of time preferences as a better descriptor of intertemporal choices, and suggest that individuals are tempted by, and some of them fall for, instantaneous gratification (e.g., Laibson, 1997). Other scholars have proposed alternative explanations. A well-known theoretical explanation is based on the role of uncertainty, which can be articulated in many ways. Yaari (1965) studies the impact of uncertain lifetime for intertemporal choices; Sozou (1995) rationalizes discounting in terms of risk that a delayed reward may not be received; Azfar (1999) studies the role of uncertain discount rates; Fernández-Villaverde and Mukherji (2006) consider random shocks on consumption preferences; Dasgupta and Maskin (2005) study an uncertain environment where payoffs may be realized early; and Halevy (2008) considers the role of uncertain lifetime and default probability. A way of dealing with both uncertainty and present-biased preferences is presented in O'Donoghue and Rabin (1999), who consider the case where the cost to complete a task is stochastic and agents may have incentives to procrastinate. Interestingly, despite the abundance of theoretical models pointing to the role of uncertainty, experimental results of intertemporal choices are often interpreted under the assumption of a certainty scenario. This assumption facilitates the identification of a point estimate for discount factors, but it neglects that uncertainty is often an intrinsic feature of intertemporal decisions, even when the experimenter does not explicitly introduce it. This may be one of the sources of the 
large variability in the estimates of discount factors reported in the literature (Frederick et al., 2002).

There exist experiments on intertemporal choices which employ money and experiments involving non-monetary rewards, such as noise (Millar and Navarick, 1984), rice (Pender, 1996), drinking water (Brown et al., 2009), and chocolate (Reuben et al., 2010). Both monetary and non-monetary domains are relevant for economic decisions and both may help in studying time preferences. The most common practice among experimental economists is to use money as a reward, which provides direct information concerning financial decisions and monetary trade-offs. It is possible, however, that the experimental results over money differ from those over consumption goods if preferences are domain-specific and/or if there are measurement errors when using monetary rewards. Hence additional evidence with non-monetary rewards can shed light on economic decisions related to consumption goods, effort exertion and completion of chores. For instance, if time preferences turned out to be domain specific, the results obtained over money rewards would not extend to very common activities in the workplace and in everyday life such as filing a tax return, tidying up the office desk, completing an assignment, going to the gym, watching TV, or playing with the children. In addition, when the stakes are non-monetary, drivers other than discounting may emerge. For example, Loewenstein (1987) argued that there may be anxiety while waiting for a bad (non monetary) outcome, and savoring while waiting for a positive (non monetary) outcome. When this is the case, one would predict behavior which goes in the opposite direction with respect to the one predicted by the Discounted Utility model. The idea behind domain-specific anticipatory feelings is appealing and intuitive, but the existing evidence over intertemporal choices is still weak (Casari and Dragone, 2011a) and deserves further experimental validation.

Furthermore, a methodological issue which may lead to measurement errors arises when the reward used in the experiment is storable and tradable outside the lab (Coller and Williams, 1999, Cubitt and Read, 2007). Storability is an issue because it does not allow us to exactly control for the timing in which the reward is consumed, which is the primitive an experimenter would like to measure. On the contrary, when the reward is to be consumed in the lab and cannot be stored for later, one can be sure about the timing of consumption and obtain more reliable information on time preferences. Tradability of money outside the lab may also bias the experimental results, because it allows for intertemporal arbitrage due to 
the existence of an external credit market $\mathrm{I}^{3}$ By employing a consumption good that cannot be stored, nor consumed outside the lab, our experiment directly addresses these issues in order to improve the reliability of the data.

\section{Experimental design}

We recruited subjects for an experiment made of three sessions over a one-month period. These experimental sessions were run at two-week intervals at dates 0,1 , and 2 . The main decision was about when to listen to a 20-minute indivisible package of annoying noise, i.e., at date 0,1 , or 2 . The sound was unpleasant but it did not cause pain. The 20-minute package of noise had to be listened to once, and it could not be spread over two or more sessions. Following participation in all three sessions, each subject received $€ 30$. Exactly 193 subjects started off the experiment: 133 under treatment 1 and 60 under treatment 2, which presents some differences in the procedure $\left.\right|^{4}$ Unless otherwise noted, the description refers to treatment 2 . We begin by describing the procedure for session 0 .

Session 0 included various color-coded parts: a ranking of the dates for noise listening (blue), an auction for flexibility (red), an auction for a dummy task (white), an auction for listening to additional noise at 0 (yellow), and a choice of exit penalty (green). In treatment 1, the actual order was white, yellow, blue, red, and green, while in treatment 2 it was white, blue, red, and green.

In the blue part, subjects expressed their preferences on when to listen to 20 minutes of noise. Before choosing, everyone listened to a 3-minute sample noise to gain familiarity with the task. This rules out curiosity for the task as a motivation for listening immediately to the noise. The available dates were 0 (today), 1 (two weeks later) and 2 (four weeks later), which generated six possible rankings (Table I). At the end of the session, a 10-face die was rolled for every participant to determine the date of noise listening, and depending on the die roll, subjects listen to the noise. If session 0 was ranked as most preferred, then the probability of listening to noise at 0 (today) was 0.60 ; the probability was 0.10 when least preferred; and 0.30 otherwise. The timing of noise listening had no impact on the monetary payments. In treatment 1 , the random draw and noise listening took place right after the

\footnotetext{
${ }^{3}$ Some consumption goods like rice or chocolate do not solve the storability and tradability issues.

${ }^{4}$ Treatment 2 also included other tasks not analysed in this paper.
} 
TABLE I. Ranking of preferred dates for noise listening, elicited in session 0.

\begin{tabular}{cccccc}
\hline \hline Rank & $\begin{array}{c}\text { Most preferred date } \\
\text { for noise listening }\end{array}$ & $\begin{array}{c}\text { Intermediate } \\
\text { preference for noise } \\
\text { listening }\end{array}$ & $\begin{array}{c}\text { Least preferred date } \\
\text { for noise listening }\end{array}$ & $\begin{array}{c}\text { No. of } \\
\text { subjects } \\
\text { (frequency) }\end{array}$ & $\begin{array}{c}\text { Demand for } \\
\text { (frequency) }\end{array}$ \\
\hline 210 & Four weeks later & Two weeks later & Today & $11(6.8 \%)$ & 3 \\
102 & Two weeks later & Today & Four weeks later & $18(11.2 \%)$ & 7 \\
012 & Today & Two weeks later & Four weeks later & $98(60.9 \%)$ & 22 \\
201 & Four weeks later & Today & Two weeks later & $5(3.1 \%)$ & 3 \\
120 & Two weeks later & Four weeks later & Today & $20(12.4 \%)$ & 7 \\
021 & Today & Four weeks later & Two weeks later & $9(5.6 \%)$ & 2 \\
\hline Total & & & & $161(100 \%)$ & $44 / 114$ \\
\hline \hline
\end{tabular}

Notes: The table lists profiles as they appeared in the instructions of treatment 2. The order in the instructions of treatment 1 was $012,102,210,021,120$, and 201 . We did not detect a bias in favor of the first profile listed in the table. Information on flexibility is available for $n=114$ subjects.

blue part. Note that there is a positive probability that the subject would listen to the noise at any date. Each subject has the incentive to truthfully reveal the ranking of noise listening for all sessions, and not to simply reveal her most preferred date, because she will benefit from selecting the highest probability for her most preferred date and the smallest probability for the least preferred one.

In the red part, participants were partitioned into groups of two and placed a bid to buy flexibility in a second-price auction. This flexibility option allows for updating the blue ranking two weeks later (date 1). The red coupons with the subjects' bids were publicly collected, randomly partitioned into pairs, and stapled. Subjects with the highest bid in their pair won the auction and paid the other bid amount in session 2. Auction results were revealed in session 1 . This auction yields a measure of the willingness to pay for flexibility. Subjects from treatment 1 who had already listened to noise in session 0 skipped the red part.

\footnotetext{
${ }^{5}$ In Treatment 1 we could not collect data on demand for flexibility from those subjects who listened to noise in session 0 . In response to the widespread preference for noise in session 0 , in Treatment 2 the decision about flexibility was anticipated to increase the number of observations.
} 
To familiarize subjects with the auction format, an auction for a dummy task (white) preceded the red part. In the white part, participants stated their minimum compensation for going to a room located one floor below the lab and bringing back a book. An experimenter publicly collected the bids, randomly partitioned and publicly stapled them in pairs. Subjects with the lowest bid in their pair won the auction and received the other bid amount at the end of the session. The results were shown on a screen, and the winners were publicly announced and immediately requested to fetch the book. The instructions explicitly mentioned the optimal strategy of revealing the true minimum compensation for the task. In treatment 1 , there were groups of four subjects.

Only treatment 1 had a yellow part with a second-price auction. Subjects bid for their willingness to listen to a 20-minute package of noise in a random group of four. The lowest bidder won the auction and listened to noise in session 0. Hence it was possible that in session 0 a subject listened twice to 20 minutes of noise, i.e., in the yellow and the blue parts. The rationale of the yellow part was to check whether noise was annoying, which it was. If a subject dropped out during a session, she received a show-up fee (€3, 1 subject did drop out). In addition, a subject could refuse to listen to the noise when asked to do so by the experimenters, i.e. to opt out. A subject that participated in all three sessions and opted out received $€ 30$ minus an exit penalty. In the green part, each subject chose her exit penalty from among $€ 15$, €20, €25, and $€ 30$. The instructions explained that raising the exit penalty could discourage opting out behavior and hence help in earning the full $€ 30$ participation fee. The exit penalty is a form of soft precommitment to listen to the noise because it does not remove the option of refusing to listen when asked to do so. Instead, it lowers the temptation of not implementing a plan of action by making it less attractive not to listen to the noise.

At the end, the winners of the white and yellow auctions were paid. In treatment 1, a non-anonymous questionnaire had to be completed before payment.

Session 1 included a ranking of the dates for noise listening (blue) and a choice over opting out (green). All subjects listened to a 1-minute sample noise $\mathrm{g}^{6}$ and then expressed their preferences on when to listen to 20 minutes of noise between date 1 (today) and 2 (two weeks later). The blue part was not completed by subjects who had listened to noise in session 0. Subjects learned the results of the auction for flexibility (red) after completing the

\footnotetext{
${ }^{6}$ Remember that in session 0 there was a 3-minute compulsory noise listening for everyone
} 
blue part. For auction winners, the relevant preferences on the date of noise listening were those revealed in session 1 ; for everyone else the relevant preferences were those revealed in session 0. A subject listened to noise in session 1 with a probability 0.66 if session 1 was ranked as most preferred, 0.33 if least preferred 7 In the green part, subjects had the option to refuse to listen to the noise if asked to do so. Finally, a 6-face die was rolled for every participant to determine the date of noise listening and, when appropriate, subjects listened to the noise.

Session 2 included a choice over opting out (green) and a questionnaire. Subjects who were not asked to listen to 20 minutes of noise in session 0 or 1 completed the green part and, when appropriate, listened to noise. All subjects then completed a questionnaire and received the participation fee net of the price paid by the winners of the auction for flexibility (red) and of the exit penalty paid by those who refused to listen to the noise.

Subjects were recruited from the undergraduate population of the University of Bologna, Italy. We conducted two waves of experiments of roughly equal size, one in 2008/2009 and another one in 2014 in response to a reviewer's request. All sessions were run on Wednesdays. In the invitation message, we asked subjects to bring reading material of their choice. In order to minimize attrition, the invitation message also specified that participation was required in all three sessions. This may have generated an oversampling of more patient subjects, but our aim is not a quantitative measure of the discount factor.

Subjects were seated at computer terminals separated by partitions. No communication among subjects was allowed. Instructions were distributed and read aloud. All decisions were taken with pen and paper. The decisions concerning the different color-coded parts were written on coupons of the same color. To listen to the noise, subjects wore high-fidelity headphones connected to the computer terminals. Including instruction reading, average session durations were 120, 120, and 75 minutes, respectively. For treatment 1 average session durations were about 75, 40, and 40 minutes, respectively. Overall, the average payment per subject was $€ 30$.

\footnotetext{
${ }^{7}$ In session 1 , any probability larger than 0.50 of listening on the most preferred date can truthfully elicit a subject's preference. Similarly, in session 0 , as long as the highest probability of implementation corresponds to the most preferred date and the lowest probability corresponds to the least preferred date, an expected utility maximizer has the correct incentives to truthfully reveal her preferences even if the probabilities of implementation differ across sessions.
} 


\section{Theoretical considerations}

In this section we present a model of intertemporal choice which allows for discounting, calendar effects, and uncertainty. We also introduce three distinct notions of choice reversal. These considerations will help in interpreting the experimental results.

\subsection{Intertemporal utility function}

Suppose that, from the perspective of date $s(s \leq t)$, the intertemporal utility $V(x, t, s)$ of consuming $x$ at date $t$ can be represented as follows

$$
V(x, t, s)=D(t-s) E_{s}\left[u\left(x_{t}\right)\right]
$$

where $D(t-s)$ is a discount function and $E_{s}\left[u\left(x_{t}\right)\right]$ is the expected instantaneous utility of consuming $x$ at $t$.

The discount function $D(t-s)$ is non negative and non increasing in the time distance between the consumption date $t$ and the decision date $s$. Aside from these requirements, the discount function can be of any form, including being exponential or hyperbolic. A strictly decreasing discount function represents an impatient decision maker, while a constant discount function for all $t$ and $s$ characterizes a perfectly patient agent.

The term $E_{s}\left[u\left(x_{t}\right)\right]$ evaluates the instantaneous utility of consuming $x$ at date $t$, given the information available at date $s$. We consider two possible factors: calendar effects and uncertainty. Calendar effects are due to calendar events which are going to surely occur at date $t$. For example, for most people the instantaneous utility of consuming their birthday cake $x$ depends on whether consumption occurs on their birthday $t$, or on another day $\tau \neq t$. Moreover, there may be random shocks that will affect utility at date $t$. The expectation operator depends on the decision date $s$ to take into account the possible impact of new relevant information which becomes available at date $s$. In a dynamic setting this may play an important role because, as in the example of the farmer making plans about which crop to plant, the new information about the weather can make the farmer change her ex-ante optimal choice. When there is no uncertainty, no calendar effects and no flow of utility-relevant information, the instantaneous utility function is stationary and simplifies to $u\left(x_{t}\right)=E_{s}\left[u\left(x_{t}\right)\right]=u(x)$ for all $s$ and $t$. 


\subsection{Static, calendar, and dynamic choice reversals}

The above drivers of intertemporal choice contribute to originate choice reversals over time. In this section we present three distinct notions of choice reversal which will be measured in our experiment. As an introduction to the formal definitions, we would like to contrast the typical choice tasks employed in the experimental and theoretical literatures on intertemporal preferences. Experimentalists generally infer choice reversal in a static setting where preferences are elicited at the same date. Subjects are presented with two decision problems: one over a short-run horizon and another over a long-run horizon. For instance, they are asked to choose between receiving $\$ 100$ now and receiving $\$ 110$ tomorrow, and between receiving $\$ 100$ in 30 days and receiving $\$ 110$ in 31 days. If a subject chooses $\$ 100$ now in the former decision and $\$ 110$ in 31 days in the latter one, then a choice reversal is detected (Frederick et al., 2002). We will call this a static choice reversal to emphasize that the choice is made at a specific point in time. Instead, most theorists, and Strotz (1955) among them, define choice reversal through comparisons within the same decision problem that is faced at different dates. Consider, for instance, a person deliberating whether to quit smoking tomorrow or the day after tomorrow. Suppose today she prefers to quit smoking starting from tomorrow rather than the following day. If tomorrow she changes her mind and chooses to delay quitting smoking to the following day, then a choice reversal is detected. We will refer to this as an instance of dynamic choice reversal 8 . Sayman and Öncüler (2009) have already remarked that most experimental designs in psychology and economics are cross-sectional rather than longitudinal.

Formally, consider two consumption goods, $x$ and $y$, and an agent facing the following decisions (see Figure I; subscripts denote the dates in which consumption is available):

Decision A: at date $s$, choice between consuming $x_{t}$ or $y_{t+1}$;

Decision B: at date $s$, choice between consuming $x_{t+1}$ or $y_{t+2}$;

Decision C: at date $s+1$, choice between consuming $x_{t+1}$ or $y_{t+2}$.

Comparing the choices made in decisions $\mathrm{A}, \mathrm{B}$, and $\mathrm{C}$, the following three notions of choice reversal can be identified.

\footnotetext{
${ }^{8}$ The same concepts are sometimes referred to as cross-sectional and longitudinal choice reversal (Sayman, and Öncüler, 2009, Read et al., 2012) or synchronic and diachronic choice reversal (Frederick et al., 2002).
} 
FIGURE I. Three intertemporal decisions.

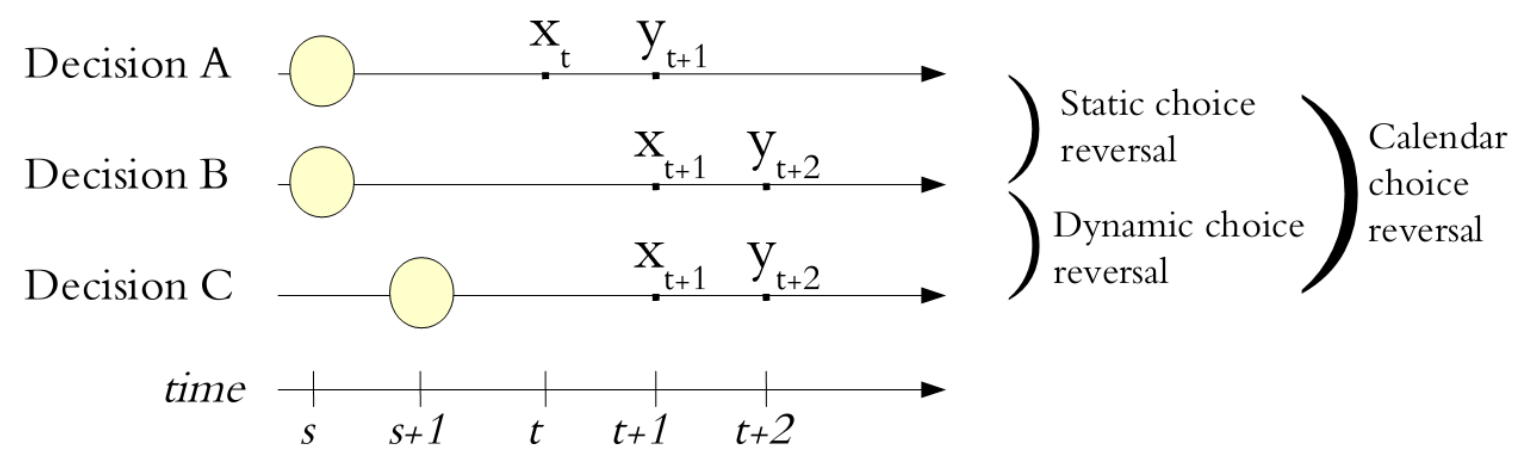

Notes: Circles denote when the choice over $x$ and $y$ is elicited. Comparison between A and $\mathrm{B}$ informs on static choice reversal, comparison between $\mathrm{B}$ and $\mathrm{C}$ on dynamic choice reversal, comparison between $\mathrm{A}$ and $\mathrm{C}$ on calendar choice reversal. In the experiment, $s=t$.

Definition 1 A static choice reversal is detected if either sooner consumption is chosen in $A$ and later consumption in $B$, or later consumption is chosen in $A$ and sooner consumption in $B$.

Eliciting intertemporal trade-offs at a single date $s$ can only detect the occurrence of static choice reversals (decisions A and B). For studying self-control problems, however, one is interested in assessing whether the choice made for a distant future (decision B) is consistent with the choice made for a closer future (decision C). In particular, when $s=t$ decision $\mathrm{C}$ involves the temptation of immediate gratification, and decision $\mathrm{B}$ represents the plan of action made when the tempting option is still distant in time. Dynamic choice reversal is formally defined as follows:

Definition 2 A dynamic choice reversal is detected if either sooner consumption is chosen in $B$ and later consumption in $C$, or later consumption is chosen in $B$ and sooner consumption in $C$.

The notion of dynamic choice reversal provides more direct evidence on self-control and temptation than the notion of static choice reversal. Moreover, the possible occurrence of dynamic choice reversal is the basic reason for demanding flexibility and precommitment, which have received much attention in the literature on self-control. We say that a decision 
maker prefers flexibility when the possibility of updating at date $s+1$ the plan made at date $s$ is valuable, from the perspective of date $s$, with respect to a rigid plan (where she cannot update her choices as time goes on). When, instead, at date $s$ an agent prefers a rigid plan over a flexible plan (because she does not want to incur a dynamic choice reversal at some date after $s$ ), she reveals a preference for precommitment..$^{9}$

Finally, a dynamic setting allows detection of a third notion of choice reversal which may occur when all calendar dates are shifted forward in time, holding constant the relative distance between decision and consumption dates. Hence, the following definition of choice reversal neatly detects the relevance of drivers for intertemporal choice other than discounting. In particular, it reveals a role for calendar effects and uncertainty in individual choices.

Definition 3 A calendar choice reversal is detected if either sooner consumption is chosen in $A$ and later consumption in $C$, or later consumption is chosen in $A$ and sooner consumption in $C$.

Static choice reversal may occur without triggering dynamic choice reversal and, analogously, detection of dynamic choice reversal is not sufficient to expect static choice reversal to occur. Detection of one type of choice reversal is sufficient to predict the occurrence of another form of choice reversal but, without specific restrictions to the intertemporal utility function (1), it is not possible to predict which one (Table II)

Under more specific assumptions, however, detection of one kind of choice reversal can provide clearer insights. Consider the textbook discounted utility model, where the instantaneous utility is stationary, there is no uncertainty, and no calendar effects (Frederick et

\footnotetext{
${ }^{9}$ Casari and Dragone (2011b) compute the value of flexibility and precommitment as functions of the individual discounting function, the expected future utility and the beliefs about the agent's future behavior.

${ }^{10} \mathrm{~A}$ subject either exhibits no choice reversal, or exhibits exactly two types of choice reversals. To see it, suppose that, without loss of generality, sooner consumption is preferred in decision $A$, and later consumption is preferred in decision $B$. This qualifies as static choice reversal. If sooner consumption is preferred in decision $C$, then there is dynamic choice reversal. If, instead, later consumption is preferred in $C$, then there is calendar choice reversal. Now consider the case where sooner consumption is preferred both in $A$ and in $B$, i.e. no static reversal is detected. If later consumption is preferred in $C$, then calendar and dynamic choice reversal are detected. If, instead, sooner consumption is also preferred in $C$, then there is no type of choice reversal.
} 
TABLE II. What can cause choice reversal.

\begin{tabular}{cccc}
\hline \hline & \multicolumn{3}{c}{ Choice reversal } \\
& Static & Dynamic & Calendar \\
\cline { 2 - 4 } Non constant discount rate & yes & yes & no \\
Calendar effects & yes & no & yes \\
Random utility shocks & yes & yes & yes \\
\hline \hline
\end{tabular}

al., 2002, Casari, 2009). Under these assumptions, the intertemporal utility of the agent of consuming $x$ at date $t$ can be written as follows:

$$
V(x, t, s)=D(t-s) u(x) .
$$

Under this specification, detection of static choice reversal is sufficient to predict dynamic choice reversal, and vice versa. Moreover, a choice reversal reveals that the discount rate is non constant. As an illustration, consider an agent who in decision $A$ prefers sooner consumption and in decision $B$ prefers later consumption (static choice reversal). In decision $C$ the agent will face the same trade-off than in decision $A$, hence she will choose sooner consumption. Since this is inconsistent with decision $B$, she will incur a dynamic choice reversal.

We conclude that employing the notion of static choice reversal as evidence suggesting that the discount rate is non constant is correct under the assumptions that uncertainty and calendar effects play no role. Otherwise, the evidence of static choice reversal is not sufficient to claim that the agent has non constant discount rates or self-control problems.

\section{Predictions}

In this section we use the model and the notions of choice reversal presented in the previous section to make predictions concerning our experimental setup. To provide benchmarks to evaluate the experimental results, we consider two models. One is the discounted utility model where, as usual, instantaneous utility is stationary and there is no uncertainty. In this model, impatience is the only driver behind intertemporal choice. Since the predictions are the same for any discounting function, including exponential and quasi-hyperbolic ones, we impose no specific restriction on its functional form and simply require strict monotonicity 
and positive discounting. The second model is a stochastic utility model: a model based on uncertainty under the assumption of perfect patience and no calendar effects. This model makes no claim to be a comprehensive model of human behavior, but it provides an additional benchmark for interpreting the experimental results. Its only purpose is to illustrate the effects of uncertainty on the revealed ranking for task completion, choice reversal, and demand for flexibility. These two models illustrate the impact of a single driver, either discounting or uncertainty, although intertemporal decisions in the field can clearly be affected by both.

In our experiment, the task to be completed is always to listen to 20 minutes of noise, regardless of the chosen consumption date $t$. Using the notation of the previous section, this implies $x=y$. According to the discounted utility model, the intertemporal utility of consuming noise $x$ at date $t$ is, from the perspective of decision date $s$ :

$$
V(x, t, s)=D(t-s) u(x) \quad(\text { discounted utility model })
$$

The predictions for such a case are presented below.

Prediction 1 (Discounted utility model). In our experiment, a subject who discounts the future under a certainty scenario with no calendar effects:

a) chooses 210 in session 0 and chooses 21 in session 1;

b) never exhibits static, dynamic and calendar choice reversal;

c) does not demand flexibility.

When the task has a fixed cost, an impatient agent always prefers to complete it at the last available date $(t=2)$. Hence, there is no static choice reversal because in session 0 the agent prefers to listen at date 2 rather than at date 1 , and at date 1 rather than at date 0 . Moreover, there is no dynamic choice reversal because the preference for postponing a costly task holds also in session 1. In other words, there is no inconsistency between choices made in date 0 and choices in date 1 . These predictions hold for the exponential discounting model as well as for the hyperbolic discounting model. In fact, the predictions are not tied to a specific functional form of discounting as long as it is monotonically decreasing in time. As the agent makes consistent choices over time and there is no uncertainty, she is indifferent between rigid and flexible planning.

The presence of 3 minutes of compulsory noise listening in session 0 for everyone and a 1 minute noise listening in session 1 reinforces the above predictions. Pilot experiments 
showed that subjects revealed a strong preference for spreading noise consumption over multiple sessions instead of listening to the entire noise-package at once. This evidence points toward a convex disutility of noise (or, equivalently a concave utility of enjoying their reading activity in silence). The best way to spread noise in the experiment is to listen to 3 , 1 , and 20 minutes in sessions 0,1 , and 2, respectively. The worst is to listen to 23,1 , and 0 minutes.

Prediction 1 also considers the case of a sophisticated subject with self-control problems who may demand precommitment to facilitate her participation in the future sessions. The show-up fee is paid only upon participation in all sessions. In our set up, precommitment takes the form of listening to the noise immediately, which increases the current cost and lowers the cost of future participation. One can prove, however, that this option is not optimal even for subjects with self-control problems.

The following predictions are based on a simple stochastic utility model. We consider an uncertainty scenario with no calendar effects where a perfectly patient agent has the following intertemporal utility function:

$$
V(x, t, s)=E_{s}\left[u\left(x_{t}\right)\right] \quad(\text { stochastic utility model) }
$$

Prediction 2 (Stochastic utility model). Consider a perfectly patient subject, an uncertainty scenario with i.i.d. shocks, and no calendar effects. In our experiment the subject:

a) prefers to complete the task immediately if the realized task cost is low enough, and to postpone it otherwise;

b) may exhibit static, dynamic, and calendar choice reversal;

c) demands flexibility.

When making her choice at date $s$, the agent knows the cost of completing the task today, and she has expectations about the cost of future completion. According to Prediction 2, the agent chooses profile 012 or 021 in session 0 if the cost of completing the task today is low, and she chooses profile 210 or 120 if the cost is high. Similarly, in session 1 she chooses profile 12 if the cost of immediate completion is low, and she chooses profile 21 if it is high $\square$

For a stochastic utility maximizer, static choice reversal may occur because, for any given choice in Decision B (Figure I), the agent facing Decision A goes sometimes for the early

\footnotetext{
${ }^{11}$ The cost of completing the task today is low if, given the current information, the utility of consuming $x$ today is higher than the (expected) utility of consuming $x$ at any other future date.
} 
option and sometimes for the later option, depending on the cost of completing the task immediately. For similar reasons, calendar and dynamic choice reversal can also occur. We conclude that, under an uncertainty scenario, choice reversals may occur even if temptation plays no role.

In our experiment, the default option is a rigid plan unless the subjects buy flexibility. Under an uncertainty scenario agents strictly prefer a flexible plan to a rigid plan because flexible planning allows them to take advantage of a favorable future realization of the cost for completing the task.

\section{Results}

This section presents four results, namely on the preferred timing for noise listening (Results 1 and 2), on choice reversals (Result 3), and on the demand for flexibility (Result 4). Exactly 193 participants initiated the experiment. Out of these, 30 subjects dropped out: 1 during session 0, 22 between session 0 and session 1, and 7 between session 1 and session 2. Moreover, 2 subjects paid the exit penalty and attended all three sessions. Our full sample is therefore made up of 161 fulfillers: subjects who attended all three sessions and listened to noise when asked to do so. Of this set of fulfillers, 71 listened to noise at date 0 . The restricted sample is made up of the remaining 90 subjects who did not listen to the noise at date 0 and therefore revealed their preferences both at dates 0 and 1 under proper incentives. Unless otherwise noted, the results refer to the full sample of fulfillers $(\mathrm{N}=161) 12$

Result 1 In session 0, about $66.5 \%$ of subjects preferred to listen immediately; less than $10 \%$ of subjects preferred to listen in four weeks.

Table I provides empirical support for Result 1. According to Prediction 1, the best choice for agents discounting the future is profile 210 , which was chosen by only $6.8 \%$ of subjects. Hence exponential and quasi-hyperbolic discounting models explain only a small fraction of the intertemporal preferences for noise listening. Result 1 does not support Prediction 1 . On the contrary, $85.7 \%$ of the rankings revealed in session 0 are compatible with a stochastic utility model (138/161 obs., Prediction 2 on rankings). A similar percentage is reported for

\footnotetext{
${ }^{12}$ In total there were 10 sessions: 7 under Treatment 1 (4 during Fall 2008 and 10 during Spring 2014) and 3 under Treatment 2 (Spring 2009).
} 
the restricted sample (74/90 obs., $82.2 \%)$. In sum, the observed choices over profiles suggest that the discounted utility model is not sufficient to predict the subjects' intertemporal choices, and that stochastic shocks may play a relevant role.

Result 1 concerns an option available "right now" vs. an option available in the future. If the present has a special status, then results concerning Decision B - where choices are made at session 0 and the rewards are available in the future (session 1 and 2) - would be different. As we show below, this is not the case.

Result 2 In session 0, about $84 \%$ of subjects preferred listening to noise in two weeks rather than in four weeks.

Support for Result 2 is in Table I. As with Result 1, Result 2 is at odds with Prediction 1 and cannot be explained by a model based on discounting as the only driver for intertemporal choices. The next result concerns choice reversal 13

Result 3 About $51 \%$ of subjects exhibited some form of choice reversal.

TABLE III. Choice reversals.

\begin{tabular}{cccc}
\hline \hline & \multicolumn{2}{c}{ Dynamic choice reversal } & Total number \\
Static choice reversal & Yes & No & of subjects \\
\hline Yes & 11 & 26 & $37(41.1 \%)$ \\
No & 9 & 44 & $53(58.9 \%)$ \\
\hline Total & $20(22.2 \%)$ & $70(77.8 \%)$ & $90(100 \%)$ \\
\hline \hline
\end{tabular}

Notes: The table includes fulfillers who did not listen to the noise in session 0 ( $\mathrm{N}=90$, restricted sample). In the whole sample of fulfillers $(\mathrm{N}=161), 52$ subjects exhibited static choice reversal. The cells on the secondary diagonal indicate that 35 out of 90 subjects exhibited calendar choice reversal.

Table III provides support for Result 3, and Table IV shows the pattern of choice reversal for each profile. When considering subjects who preferred to anticipate noise listening (i.e.

\footnotetext{
${ }^{13}$ Static choice reversal occurs if one chooses either 021, 021, 120, or 102. Dynamic choice reversal occurs if one chooses $012,102,120$ in session 0 and then 21 in session 1, or 210, 201, 021 in session 0 and then 12 in session 1. Calendar choice reversal occurs if either 012, 021 or 201 is chosen in session 1 and then 21 in session 2, or 210, 120, or 102 in session 1 and then 12 in session 2 .
} 
profile 012), the majority significantly showed a good degree of consistency and exhibited no static, dynamic nor calendar choice reversal (binomial test, proportion of dynamic and calendar choice reversal $<30 \%, p<.01, \mathrm{~N}=42$ ). Analogously, subjects preferring to postpone noise listening (profile 210) tended to exhibit no choice reversal. Overall, about half of the subjects fell in these two categories, while the remaining half exhibited some form of choice reversal. In line with Result 1, in session 1 about $73 \%$ of the subjects preferred to listen immediately (Decision C).

TABLE IV. Choice reversal by preference profile

\begin{tabular}{|c|c|c|c|c|c|}
\hline \multirow{2}{*}{$\begin{array}{l}\text { Ranking in } \\
\text { session } 0\end{array}$} & \multicolumn{4}{|c|}{ Ranking in session 1} & \multirow[t]{2}{*}{ Total } \\
\hline & & 21 & & 12 & \\
\hline 012 & $6(14.3 \%)$ & dyn, cal & $36(85.7 \%)$ & no choice rev. & 42 \\
\hline 021 & $1(25 \%)$ & stat, cal & $3(75 \%)$ & stat, dyn & 4 \\
\hline 102 & $3(23 \%)$ & stat, dyn & $10(76.9 \%)$ & stat, cal & 13 \\
\hline 120 & $4(23.5 \%)$ & stat, dyn & $13(76.5 \%)$ & stat, cal & 17 \\
\hline 201 & $2(66.7 \%)$ & stat, cal & $1(33.3 \%)$ & stat, dyn & 3 \\
\hline 210 & $8(72.7 \%)$ & no choice rev. & $3(27.3 \%)$ & dyn, cal & 11 \\
\hline Total & $24(27 \%)$ & & $66(73 \%)$ & & 90 \\
\hline
\end{tabular}

When considering the restricted sample, about $41.1 \%$ of subjects exhibited static choice reversal, $38.9 \%$ exhibited calendar choice reversal, and $22.2 \%$ exhibited dynamic choice reversal. In our setup, both exponential and present-biased individuals should exhibit no choice reversal (Prediction 1). The widespread presence of choice reversal contradicts Prediction 1. and points toward a fundamental role of other factors beyond present-biased preferences (and, more in general, discounted utility models). A model of stochastic utility is one possible candidate, as it is compatible with the observed preference profiles and the patterns of choice reversals (Prediction 2).

Static and calendar choice reversals are empirically found to occur with similar frequencies. A Pearson $\chi^{2}$ test indicates that there is a statistically significant relationship between those exhibiting static and calendar choice reversal: 26/37 of the subjects exhibiting static choice reversal also exhibit calendar choice reversal, while $9 / 53$ subjects exhibiting no static choice reversal also exhibit calendar choice reversal (70.3\% vs. 17\%, Pearson 
$\left.\chi^{2}(1)=26.04, p<0.001, \mathrm{~N}=90\right)$. In particular, evidence of calendar choice reversal calls for a role for exogenous shocks on choices concerning completing the task today versus completing it in two weeks.

Below we present the results on the demand for flexibility, which are based on the choices of 114 subjects.

Result 4 About $38 \%$ of subjects were willing to pay for flexibility.

Positive bids in the auction for flexibility (red part) indicate a demand for flexibility. In a certainty scenario, neither exponential nor quasi-hyperbolic discounters should demand flexibility (Predictions 1). On the contrary, flexibility is valuable in an uncertainty scenario where the task cost can change depending on stochastic shocks. We report a higher demand for flexibility among subjects who exhibited some form of choice reversal. There are 22 subjects who demanded flexibility out of the 46 who reversed their choices, and 13 subjects who demanded flexibility out of the 44 who never reversed their choices ( $47.8 \%$ vs. $29.6 \%$, Pearson $\left.\chi^{2}(1)=3.16, p=0.075, \mathrm{~N}=90\right)$. While the demand for flexibility is an ex-ante measure of uncertainty, calendar choice reversal best captures the ex-post impact of exogenous shocks at decision time. In the data, there is no significant positive correlation among the two measures: there are 17 subjects who demanded flexibility out of the 35 who exhibited calendar choice reversal, and 18 out of the 55 who did not reverse their choices (48.6\% vs. $32.7 \%$, Pearson $\left.\chi^{2}(1)=2.26, p=0.13, \mathrm{~N}=90\right)$. A model of stochastic utility is compatible with these patterns of behavior

Our results do not seem an artefact of the lack of economic training, as the subjects with Humanities majors were more likely to behave according to the discounting model (Prediction 1) than the subjects with an Economics major. Regressions on individual characteristics show that gender did not play a significant role in any of the above choices (except that males are less likely to demand flexibility), while in some of them cognitive abilities, academic major, and smoking habits played a role. Consistent with the fact that the noise was annoying, subjects who had already listened to the additional noise in Treatment 1 (yellow part with the second price auction) were more likely to choose listening now as their worst option ( $36.7 \%$ vs. $21 \%$, Pearson $\left.\chi^{2}(1)=2.86, p=0.09, \mathrm{~N}=111\right)$.

\footnotetext{
${ }^{14}$ Only $24.6 \%$ (28/114) increased the exit penalty. This evidence does not contradict Predictions 1 and 2 , although no quantitative prediction could be made. Half of the 114 subjects demanded neither flexibility nor increased the exit penalty.
} 


\section{Discussion}

The reported evidence deserves some comments. First, in the experiment the canonical models of exponential or quasi-hyperbolic discounting lack descriptive power (Prediction 1). In the experiment only 6 out of 114 subjects fit all predictions of the discounted model: this result is stunning, as it amounts to about $5 \%$ of the observations. According to the discounted utility model, the best option for noise listening is the latest available date and the worst option is the earliest available date, so there should be no demand for flexibility and no choice reversal of any kind. Contrary to Prediction 1, however, a large majority of subjects chose to anticipate noise listening and to avoid doing it at the last available date, and about half of the subjects reversed their choices according to at least one of our definitions 15

Recall that, in this experiment, a single-drive model based only on discounting predicts no choice reversal. This prediction holds for any discounting function, and is shared by both exponential and present-biased agents. The explanation for the fact that choice reversal does occur, then, must rely on some motivation other than impatience and temptation. As an instance of a possible candidate, we explore the role of uncertainty about the cost of completing the task, and we report promising findings. Three cues reveal a key role for uncertainty. One is demand for flexibility, which is direct evidence that the subject fears future shocks. The other two are dynamic and calendar choice reversal, which may occur in case of unpredicted events. In the restricted sample, 48 out of 90 subjects either demanded flexibility or displayed dynamic choice reversal (53.3\%), which points toward a substantive impact of uncertainty in intertemporal choices. A model of stochastic utility can go a long way in explaining the data because it can encompass anticipation of noise listening, choice reversal, and demand for flexibility. The drawback is that it is more difficult to falsify because it is compatible with a larger set of behavioral patterns than a discounting model $[16$ However, this exercise is useful because it shows an avenue for future research with the potential to match the empirical evidence with the theoretical models on intertemporal choice.

\footnotetext{
${ }^{15}$ Modifying the textbook (additively separable) discounted utility model would produce different predictions and possibly allow for some form of choice reversal. In this sense, our evidence reinforces the notion that the textbook model that is commonly used in the literature has desirable analytical properties, but a limited descriptive power.

${ }^{16}$ As an example, see Casari and Dragone (2001b).
} 
Second, we provide new, incentivized evidence on a real effort task that is complementary to the existing studies based on monetary rewards. Moreover, our design has the methodological advantage of addressing serious concerns related to the storability and tradability of monetary rewards (Cubitt and Read, 2007) and to exhibit predictions that do not depend on the concavity of the instantaneous utility function, nor on the shape of the discounting function. Non-monetary choices are very relevant for a wide array of field decisions, and there exists a rich theoretical literature about non-monetary choices (e.g. O'Donogue and Rabin, 1999). Yet, experiments with this feature are rare in the economic literature (for an exception concerning real effort, see Falk and Ichino, 2006). One may also think that anticipatory feelings may contribute to explain intertemporal choices in the experiment. If subjects experience dread or anxiety while waiting for the moment of noise listening, anticipating the date of listening may be optimal, which would account for one major pattern in the data. On the other hand, under a certainty scenario subjects with anticipatory feelings should be dynamically consistent and exhibit no sort of choice reversal. As a consequence, subjects with anticipatory feelings should not demand flexibility. Overall, when confronted with the data, a simple model of anticipatory feelings accounts for about one third of the individual choices elicited at date $0(29 / 90=32.2 \%$ of subjects $)$.

A final issue is related to the stimulus used in the experiment. Since the experimental setup concerns a specific real effort task which confronts subjects with choices over losses, it is legitimate to wonder whether our results would also apply when considering different tasks, or different domains of choice. Since some recent evidence based on hypothetical questions suggests that people discount losses and gains asymmetrically (see, e.g., Abdellaoui et al., 2010, Hardisty et al., 2012, Appelt et al., 2013), would the relative importance of discounting and uncertainty be different if we had considered, say, scheduling a massage, rather than listening to noise? Although interesting, this is not the research question of this paper. Unlike other studies, our aim is not a calibration of the discount factor, nor the exact assessment of the quantitative impact of the various drivers behind intertemporal choices. Our goal is to neatly show the empirical relevance of motivations other than discounting, and to report clean evidence on choice reversal occurring without being triggered by temptation. We leave to further experimental studies the investigation of other tasks and rewards, and to assess whether the sign and magnitude of the reported findings may generalize to other experimental scenarios. 


\section{Conclusions}

Empirical studies on time preferences are methodologically difficult because of the intermingling of various motivations driving individual behavior. The main focus of the literature has been obtaining quantitative estimates of the discounting function and disentangling exponential discounting from quasi-hyperbolic discounting. This study takes a different perspective and aims to uncover other motivations that differ from impatience and temptation but that can still be relevant for intertemporal choice.

We elicited a subject's plan concerning the preferred date for completing a given task, and we then tracked its implementation over time. The experimental design had a multi-stage structure because subjects were required to make their decisions at multiple dates. This dynamic design is more informative than the static design often used in the experimental literature on time preferences. Not only does it allow us to directly observe individual behavior over time, but it also allows for a deeper understanding of time-inconsistent behavior. In particular, one can detect three different notions of choice reversal: static, dynamic and calendar. These notions are complementary and each of them sheds light on different driving forces behind intertemporal choice. Static choice reversal compares choices in the short-run vs. the long-run horizon. Most experimental literature relies on evidence based on static choice reversal when testing models of intertemporal choice, although this evidence can actually inform us about the possible occurrence of self-control problems only under specific assumptions. A more direct measure of self-control problems is given by the detection of dynamic choice reversal, because this reveals the existence of possible differences between planned and implemented behavior. This dynamic notion captures the argument made by Strotz (1955) on time inconsistency and the relevance of the temptation of immediate gratification in self-control issues (Laibson, 1997). Finally, a calendar choice reversal can be triggered by events such as birthdays and it is independent from how the agent discounts the future. In such a reversal, a subject switches choice if the whole decisional block (elicitation and delivery dates) shifts forward in terms of calendar dates. This paper presents empirical measures of all types of choice reversal, in particular of dynamic and calendar ones.

The reported evidence can hardly be explained by exponential and quasi-hyperbolic dis-

counting alone. About two thirds of the subjects preferred to complete the costly task immediately, rather than postponing it to the last available date. About half of the subjects 
exhibited static, dynamic or calendar choice reversal. Overall, only $5 \%$ of the subjects fit the predictions of a model of intertemporal choice based only on discounted utility. Given that the role of impatience and temptation in intertemporal choices has already been convincingly documented in other empirical studies, the data suggest that, besides discounting, there are other drivers for intertemporal choices. In particular, the uncertainty about future utility most likely plays a substantial role.

This study points toward two avenues for future research in intertemporal choices. First, employing a dynamic setup allows for a deeper understanding of time preferences and time consistency. A second avenue is studying how the most common types of uncertainties affect intertemporal choices and the consequent policy implications to improve self-control. Here, we focus on the scheduling of a costly task to be completed within a deadline and we have shown that the canonical exponential discounting model or the quasi-hyperbolic discounting model cannot explain the empirical evidence. This result clearly calls for models of intertemporal choice that allow for a wider range of motivations, in which individual preferences are not only affected by impatience and temptation, but also by other factors such as calendar effects and uncertainty.

\section{References}

[1] Abdellaoui, M., Attema, A. E., and Bleichrodt, H. (2010). "Intertemporal Tradeoffs for Gains and Losses: An Experimental Measurement of Discounted Utility" . The Economic Journal, 120, 845-866.

[2] Ainslie, G., and Haendel, V. (1983). "The Motives of the Will". In E. Gottheil, K. A. Druley, T. E. Skodola, \& H. M. Waxman (Eds.), Etiologic aspects of alcohol and drug abuse. Springfield: Charles C. Thomas

[3] Appelt, K. C., Hardisty, D. J., and Weber, E. U. (2011). "Asymmetric Discounting of Gains and Losses: A Query Theory Account". Journal of Risk and Uncertainty, 43, 107-126.

[4] Ariely, D., and Wertenbroch, K. (2002). "Procrastination, Deadlines, and Performance: Self-control by Precommitment". Psychological Science, 13, 219-224. 
[5] Azfar, O. (1999). "Rationalizing Hyperbolic Discounting." Journal of Economic Behavior and Organization, 38, 245-252.

[6] Benartzi, S., and Thaler, R. H. (2004). "Save More Tomorrow: Using Behavioral Economics to Increase Employee Saving." Journal of Political Economy, 112, S164-S187.

[7] Benhabib, J., Bisin, A., and Schotter, A. (2006). Present-Bias, Quasi-Hyperbolic Discounting, and Fixed Costs, working paper, New York University

[8] Brown, A. L., Chua Z. E., and Camerer, C. F. (2009). "Learning and Visceral Temptation in Dynamic Saving Experiments." The Quarterly Journal of Economics, 124, $197-223$.

[9] Casari, M. (2009). "Precommitment and Flexibility in a Time-decision Experiment." Journal of Risk and Uncertainty, 38, 117-141.

[10] Casari, M., and Dragone, D. (2011a). "On Negative Time Preferences." Economics Letters, 111, 37-39.

[11] Casari, M., and Dragone, D. (2011b). "Impatience, Anticipatory Feelings and Uncertainty: A Dynamic Experiment on Time Preferences." wp DSE 777, University of Bologna.

[12] Charness, G., and Gneezy, U. (2009). "Incentives to Exercise." Econometrica, 77, 909931.

[13] Coller, M., and Williams, M. B. (1999). "Eliciting Individual Discount Rates". Experimental Economics, 2, 107-127.

[14] Cubitt, R. P., and Read, D. (2007). "Can Intertemporal Choice Experiments Elicit Time Preferences for Consumption?" Experimental Economics 10, 369-389

[15] Dasgupta, P., and Maskin, E. (2005). "Uncertainty and Hyperbolic Discounting." American Economic Review, 95, 1290-1299.

[16] Dellavigna, S., and Malmendier, U. (2006) "Paying Not To go To The Gym." American Economic Review, 96, 694-719. 
[17] Falk, A., and Ichino, A. (2006). "Clean Evidence on Peer Effects". Journal of Labor Economics, 24, 39-58.

[18] Frederick, S., Loewenstein, G., and O'Donoghue, T. (2002). "Time Discounting and Time Preference: A Critical Review." Journal of Economic Literature, 15, 351-401.

[19] Fundenberg, D., and Levine, D. K. (2006). "A Dual Model of Impulse Control." American Economic Review, 96, 1449-1476.

[20] Ginè, X., Goldberg, J., Silverman, D., and Yang, D. (2012). "Revising Commitments: Field Evidence on the Adjustment of Prior Choices." NBER Working Paper No. 18065.

[21] Halevy, Y. (2008). "Strotz meets Allais: Diminishing impatience and the certainty effect." American Economic Review, 98, 1145-1162.

[22] Hardisty, D. J., Appelt, K. C., and Weber, E. U. (2013). "Good or Bad, We Want it Now: Fixed-cost Present Bias for Gains and Losses Explains Magnitude Asymmetries in Intertemporal Choice". Journal of Behavioral Decision Making, 26, 348-361.

[23] Kirby, K. N., and Herrnstein, R.J. (1995). "Preference Reversals due to Myopic Discounting of Delayed Reward." Psychological Science, 6, 83-89.

[24] Laibson, D. (1997). "Golden Eggs and Hyperbolic Discounting." Quarterly Journal of Economics, 112, 443-77.

[25] Loewenstein, G. (1987). "Anticipation and the Valuation of Delayed Consumption." Economic Journal, 97, 666-84.

[26] Loewenstein, G., and Prelec, D. (1991). "Negative Time Preference." American Economic Review, 81, 347-352.

[27] Manzini, P., and Mariotti, M. (2010). "Moody Choice." IZA Discussion Paper 5005.

[28] Millar, A., and Navarick, D.J. (1984). "Self-control and Choice in Humans: Effects of Video Game Playing as a Positive Reinforcer." Learning and Motivation, 15, 203-218.

[29] O'Donoghue, T., and Rabin, M. (1999). "Incentives for Procrastinators." Quarterly Journal of Economics, 114, 769-816. 
[30] Pender, J. L. (1996). "Discount Rates and Credit Markets: Theory and Evidence from Rural India." Journal of Development Economics, 50, 257-296.

[31] Read, D., Frederick, S., and Airoldi, M. (2012). "Four Days Later in Cincinnati: Longitudinal Tests of Hyperbolic Discounting". Acta Psychologica, 140, 177-185.

[32] Read, D., and van Leeuwen, B. (1998). "Predicting Hunger: The Effects of Appetite and Delay on Choice." Organizational Behavior and Human Decision Processes, 76, 189-205.

[33] Read, D., Loewenstein, G., and Kalyanaraman, S. (1999). "Mixing Virtue and Vice: Combining the Immediacy Effect and the Diversification Heuristic". Journal of Behavioral Decision Making, 12, 257-273.

[34] Reuben, E., Sapienza, P., and Zingales, L. (2010). "Time Discounting for Primary and Monetary Rewards." Economics Letters, 106, 125-127

[35] Samuelson, P. A. (1937). "A Note on Measurement of Utility." The Review of Economic Studies, 4, 155-161.

[36] Sayman, S., and Öncüler, A. (2009). "An Investigation of Time Inconsistency". Management Science, 55, 470-482.

[37] Schelling, T. C. (1978). "Egonomics, or the Art of Self-Management." American Economic Review, 68, 290-294.

[38] Sozou, P. D. (1998). "On Hyperbolic Discounting and Uncertain Hazard Rates." Proceedings of the Royal Society of London: Biological Sciences (Series B), 265 (1409), 2015-2020.

[39] Strotz, R. H. (1955). "Myopia and Inconsistency in Dynamic Utility Maximization." Review of Economic Studies, 23, 165-80.

[40] Tanaka, T., Camerer, C., and Nguyen, Q. (2010). "Risk and Time Preferences: Linking Experimental and Household Survey Data from Vietnam." American Economic Review, $100,557-571$. 
[41] Warner, J. T. and Pleeter, S. (2001). "The Personal Discount Rate: Evidence from Military Downsizing Programs." American Economic Review, 91, 33-53.

[42] Yaari, M. (1965). "Uncertain Lifetime, Life Insurance, and the Theory of the Consumer." Review of Economic Studies, 32, 137-150. 